\title{
The Influence of Robot Number on Robot Group Perception-A Call for Action
}

\author{
RICARDA WULLENKORD and FRIEDERIKE EYSSEL, Bielefeld University
}

\begin{abstract}
Research on robot groups has often applied psychological principles underlying group processes between humans to interactions with and between robots. However, such research has failed to test empirically whether these principles indeed apply to the robot context. For instance, the notion of a social group may be interpreted differently when facing human versus robot groups. Basic research on this issue is missing. Therefore, the present experiment aimed at integrating social psychological theorizing and research on robot groups by utilizing the principles of group entitativity. We examined the effect of robot number and similarity on the perception of these robots as a (social) group. To do so, participants saw pictures of one to ten robots, appearing low or high in similarity. Results showed that the aspects eliciting the perception of a social "group" in humans seem to differ from the factors evoking robot group perception. According to our findings, at least three robots seem necessary for the perception of a robot "group" to emerge. Social psychological research, however, has proposed that two persons suffice to elicit the notion of a human social group. Basic research is needed to substantiate assumptions drawn from social psychological theorizing before translating it into human-robot context.
\end{abstract}

CCS Concepts: • Applied computing $\rightarrow$ Psychology; • Human-centered computing $\rightarrow$ HCI theory, concepts and models;

Additional Key Words and Phrases: Robot groups, group perception, entitativity

ACM Reference format:

Ricarda Wullenkord and Friederike Eyssel. 2020. The Influence of Robot Number on Robot Group PerceptionA Call for Action. ACM Trans. Hum.-Robot Interact. 9, 4, Article 27 (July 2020), 14 pages.

https://doi.org/10.1145/3394899

\section{INTRODUCTION}

Imagine a future in which robots are an integral part of everyday life. Think of an everyday life scenario that includes such technology. How many robots would be part of it? For many people, such a scenario may include more than just one robot. This notion is not far-fetched, given the current advancements, e.g., in the field of assistance technologies and the practice in industrial settings [1]. One promising sector for robotic assistance, of one or even several robots at once, is that of nursing homes where robots are deployed to support caretakers by offering service to

This research was supported by the Cluster of Excellence Cognitive Interaction Technology "CITEC” (EXC 277) at Bielefeld University, which is funded by the German Research Foundation (DFG).

This research was funded by the Ministry of Education and Research (Project "poliTE"; grant no. 16SV7880K).

Authors' address: R. Wullenkord and F. Eyssel, CITEC, Inspiration 1, 33619 Bielefeld, Germany; emails: rwullenk@citec.uni-bielefeld.de, feyssel@uni-bielefeld.de.

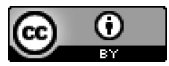

This work is licensed under a Creative Commons Attribution International 4.0 License.

(C) 2020 Copyright held by the owner/author(s). Publication rights licensed to ACM.

2573-9522/2020/07-ART27

https://doi.org/10.1145/3394899 
elderly people or by monitoring their health status [2]. Cognitive rehabilitation of seniors with mild cognitive impairments constitutes another domain where robot assistance could be useful [3]. However, having to interact with multiple robots simultaneously might have strong implications for the perception of these robots and for users' reactions toward them. For example, results of social psychological research on human groups (e.g., Reference [4]) have suggested that seeing a robot as an outgroup member rather than an ingroup member changes cognition, affect, and behavior toward it. Furthermore, people seem to behave more competitively within human-human intergroup contexts compared to interindividual interactions [5]. This might likely be the same for robots. One of the most influential forces in human person and group perception-social categorization (e.g., Reference [6])-might drive such effects: The perception of others as members of various (social) groups is a key factor in human social cognition (e.g., Reference [7]). Social categorization, i.e., the process of categorizing people into groups based on their characteristics (i.e., age, gender), allows us to simplify information processing. Thanks to social categorization, we do not have to process every single piece of information available, but at the same time, social categorization influences perception and behavior. Therefore, understanding the perception of robots as social agents and parts of (social) groups is key for understanding and predicting users' reactions toward them.

\section{RELATED WORK}

Knowledge about the effects of group membership on human behavior and the perception of, and the behavior toward, groups of human beings (e.g., References [8-10]) is well established in the domain of psychology. For nonhuman entities, this is not yet the case. However, the systematic investigation of the notion of robot groups from a social psychological point of view has become a topic of scientific inquiry recently (e.g., References [11-15]). Previous research has explored, among other things, conformity toward robot groups [11-13] and the influence of robot groups on user behavior and emotions [14-16]. Some studies implied that mechanisms of social categorization and group perception are similar between groups of robots and humans. For instance, a robot that was identified as part of the participants' ingroup was perceived more positively and participants were more willing to interact with it compared to a robot that was not part of the ingroup [17, 18]. These effects match classic findings in human-human intergroup contexts $[17,18]$. Other studies, in contrast, showed rather inconclusive findings: Participants rated a robot with a different ethnic background more positively compared to a robot with the same ethnic background as the participant [19]. For human beings, the expectation is the exact opposite. This raises questions regarding the perception of robots as a group, in comparison to human group perception. A precondition for effectively deploying robots in society and for understanding and predicting user reactions is the knowledge about the boundary conditions for the perception of a given number of robots in terms of a "group." This is important when trying to understand user behavior toward robots, as behavior toward individuals differs from behavior toward groups [5] in that people act much more aggressively and less cooperatively in intergroup situations compared to interindividual situations (e.g., References [20-22]). Thus, it must be systematically determined to what extent groups of humans and robots are similar and to what extent they differ. What makes people perceive a given number of robots as a group? What makes a robot group a "social" group? Moreover, do the psychological mechanisms that are at work in human intergroup contexts translate to robot intergroup contexts?

Lickel et al. [23] have postulated several factors that influence entitativity [24], which is defined as the extent to which a group is perceived as a coherent social entity, from members as well as non-members. The factors postulated by Lickel and colleagues [23] are group size, similarity, permeability of group borders, duration of the group, interdependence between/interaction 
of group members, and the importance of the group for the members. Following Lickel et al. [23], the factors influence group entitativity to varying degrees, with interaction, importance, interdependence, and similarity being most closely related to group entitativity.

Social psychological research suggests that two human beings sharing characteristics (like a goal) are sufficient in number to evoke the perception of them as a group [25, 26], with Reference [26] providing an overview over several different definitions of groups. This assumption is mostly based on theoretical reasoning (e.g., References [27, 28]) and field observations (e.g., Reference [29]). For robots, the minimal number leading to group perception of robot individuals is unknown. Different experiments that have examined robot groups have used varying numbers of robots; for example, two [30] or three robots [31] to represent a "group" of robots. However, when the goal is to study robot group perception, it is crucial to know the exact number of robots required to elicit a sense of an entitative group. To shed light on this issue, the present study focuses on two factors: group size and similarity. Group size is important, as studies examining robot groups have used differing group sizes when examining robot groups. Similarity should be studied, as this aspect plays a key role for group entitativity. The perception of groups of humans and groups of robots seems to differ to some extent; hence, the existing studies (e.g., References [12, $15,30,31]$ ) have built on the knowledge about human groups without scrutinizing whether these claims hold true for robots as well. Possibly, two or three robots are not sufficient in number to elicit the perception of these robots as a group; that is, they might still be perceived as individual robot prototypes, resulting in differences in user perceptions of and behavior toward these robots. To illustrate, behavior toward a group of robots would presumably be more competitive compared to individual robots, considering the findings from human group research [5]. Additionally, whether a group is perceived as entitative or not influences processing of information given by the group [32, 33]. Furthermore, some of the studies have used several of the same robots [12], while others have used different robots [15]. It remains unknown whether the similarity of the robots influences robot group perception: Three robots high in similarity might be perceived as a group, whereas presenting three robots low in similarity might not lead to group perception. The present study aimed at elucidating this basic research question further to pave the way for subsequent research on robot groups. Our work addressed two main research questions: (1) How many robots are necessary to lead to group perception?; and (2) how does similarity between robot types influence this perception? Additionally, we were interested in the effects of robot group number on negative ratings of robots, e.g., robot anxiety, perceived threat, negative attitudes toward robots, or reduced mind perception, as previous research has shown that a larger number of robots was perceived as more threatening [15] and that group members, especially those high in perceived entitativity, were perceived to have less mind [34]. We included measures of group perception and a manipulation check on entitativity, as well as measures capturing attitudes and anxiety toward robots. Regarding Research Question 1, we were interested in exploring whether the number of robots that leads to group perception is similar compared to what was found in human context, namely, two, or if a higher number of robots is necessary to lead to group perception, and what effect the number of robots has on perceived group entitativity. Hypothesis 1 predicted that high similarity would lead to stronger group perception and more perceived group entitativity than low similarity. According to Hypothesis 2, when confronted with a higher number of robots, participants should rate these robots more negatively. More specifically, they should report more perceived threat, more negative attitudes toward robots, more robot anxiety, and less mind attribution toward robots. Reference [23] used a text-based approach to manipulate group entitativity, whereas References [30] and [31] have used a visual approach. A visual approach may be closer to a "real world" experience, due to the fact that people will more probably see robots than read about them. Therefore, we decided to use a visual approach in this study as well. 


\section{METHOD}

\subsection{Design and Sample}

The online study was designed as a $2 \times 10$ between-subjects design. Similarity of the robots (low vs. high) and number of robots ( 1 to 10 ) were varied between participants, resulting in 20 conditions. The sample consisted of 425 people. Most of the participants $(n=317)$ were students. One participant's data were excluded from data analyses due to produced outlier scores on more than $5 \%$ of the variables. This participant had also reported extensive HRI experience and markedly differed from the remaining participants in that respect as well. An outlier score was defined as a value more than three standard deviations above or below the mean.

Of the remaining participants, 136 were male, 276 were female, 8 people were of other gender, and 4 participants did not indicate their gender. The sample ranged in age between 17 and 60 years $\left(M_{\text {age }}=25.68, S D_{\text {age }}=7.41\right)$. Ten participants did not indicate their age.

\subsection{Procedure}

The experiment was carried out online using Unipark software by Questback. Participants were recruited via posting the link in social networks and forums as well as by posting and distributing flyers. The completion of the questionnaire took about five to ten minutes. In the beginning, participants were informed about the purpose of the study, gave informed consent, and started the questionnaire. Each participant was presented with a picture (sized $7.87 \times 4.33$ inches) that featured either one robot or a picture of several robots that appeared either low or high in similarity. The poses for the robots high in similarity were always identical, the poses for the robots low in similarity were different. Moreover, there were slight differences in the coloring of the robots low in similarity; that is, there were up to three different shades of blue. Figure 1 shows an example picture of two robots high in similarity showing identical poses. We used identical images for the robots, as they were perceived as being highly similar to each other.
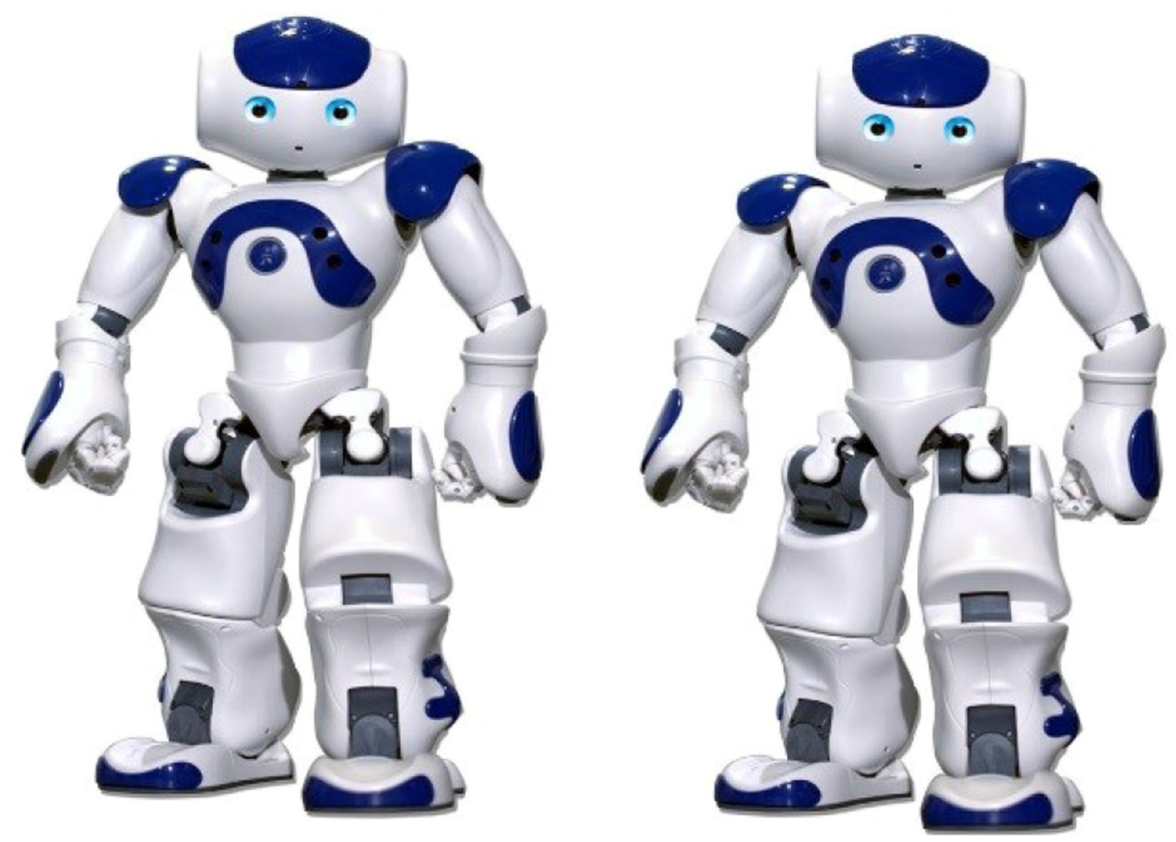

Fig. 1. Stimulus material used in the experiment. Two robots in identical poses with identical coloring. 

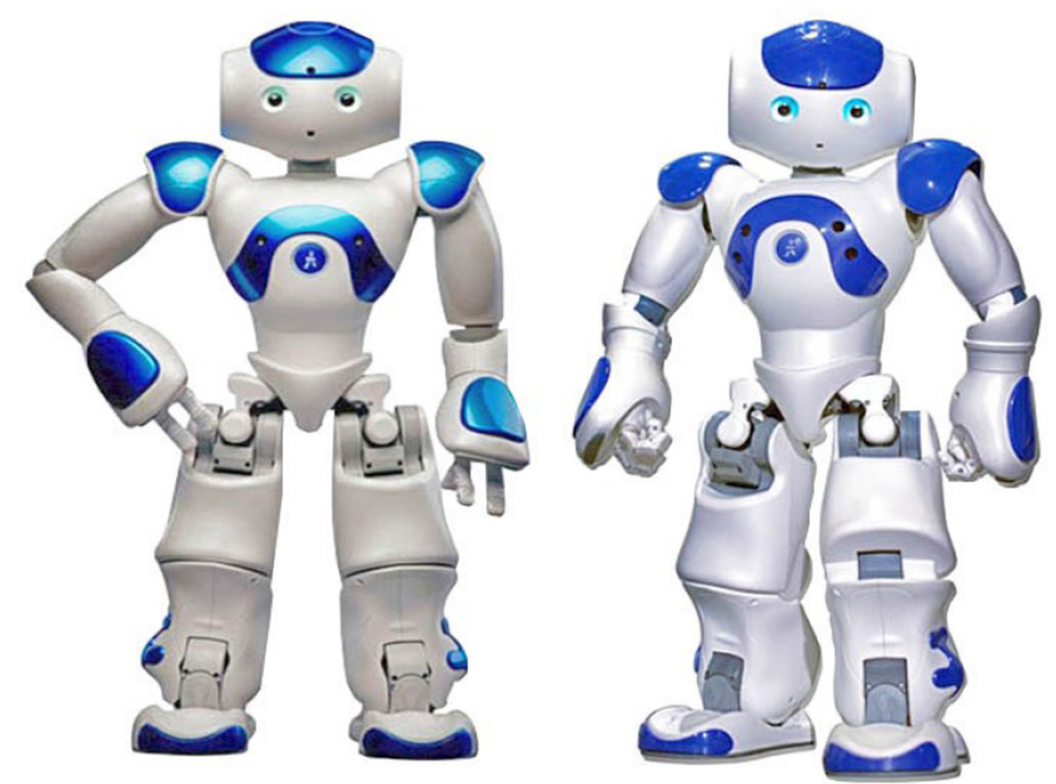

Fig. 2. Stimulus material used in the experiment. Two robots in different poses with different coloring.

Figure 2 depicts a stimulus with two robots low in similarity; that is, these two robots feature different poses and slightly different coloring.

Participants were asked to closely inspect the picture. We did not randomize robot poses for participants in the low-similarity conditions; participants belonging to the same experimental condition saw identical images. Afterwards, they proceeded with the questionnaire.

\subsection{Dependent Measures}

If not declared otherwise, responses were provided using 7-point Likert scales, with 1 indicating low endorsement, and 7 indicating high endorsement of the measured construct, respectively.

First, participants were asked to answer three items regarding how much they perceived the displayed robots as a group and how threatening they rated them ("To what extent do you perceive the displayed robots as a group?"; "Do you perceive the robots at display more as individuals or more as a group?"; "How threatening do you perceive the robots at display?"). The following six items measured perceived group entitativity of the robots (see Reference [35]). An example item from Reference [35] reads: "This group has an organized structure." Subsequently, participants responded to five questions regarding groups of robots in general, e.g., "Robots, as humans, can form a group." Afterwards, we administered 12 questions on acceptance and usage of new technologies (TA, Reference [36]). "I am curious toward new technical developments" is one of these items. This was followed by the Negative Attitudes toward Robots Scale (NARS, [37]), which consists of 15 items, and the 10-item Robot Anxiety Scale (RAS, Reference [38]). An example item of the NARS reads, "I would be nervous if I had to operate a robot in front of other people." The RAS consists of items such as, "I am worried about how fast a robot would move."

Finally, participants reported mind attribution to robots with a short 10-item version of the Mind Attribution Scale (MAS, Reference [39]); e.g., "To what extent are robots able to experience joy?". After that, they completed six questions regarding their prior robot experience (e.g., Reference [40]); for example, "Did you already participate in a study with this robot?". This was followed 
by demographic questions. Finally, participants had the opportunity to get course credit for their participation, were thanked, and debriefed.

\section{RESULTS}

\subsection{Preliminary Analyses}

Means, standard deviations, and internal consistencies were computed. Internal consistencies are reported using Cronbach's $\alpha$. All scales showed good reliability: they ranged from $\alpha=.82$ for MAS, perceived threat and robot group perception to $\alpha=.88$ for NARS. These descriptive statistics are depicted in Table 1.

Table 1. Descriptive Statistics and Internal Consistencies for Dependent Measures

\begin{tabular}{lccc}
\hline Scale & $M$ & $S D$ & Cronbach's $\alpha$ \\
\hline Entitativity & 4.02 & 1.31 & .86 \\
NARS & 3.79 & 1.04 & .88 \\
RAS & 3.60 & 1.15 & .86 \\
MAS & 2.81 & 0.95 & .82 \\
Threat & 4.09 & 1.49 & .82 \\
Group perception & 4.62 & 1.90 & .82 \\
TA & 3.93 & 0.64 & .83 \\
\hline
\end{tabular}

\subsection{Main Analyses}

Research Question 1 aimed to examine whether two robots (vs. a higher number) would be sufficient for group perception to emerge, and in what way the given number of robots affects perceived group entitativity. Hypothesis 1 predicted an effect of similarity between the robots on group perception and group entitativity. Robots high in similarity should be perceived as higher in entitativity and should be perceived more like a group than robots low in similarity. Additionally, we were interested in the effects of similarity and robot number on attitudes toward robots, robot anxiety, and perceived threat. Accordingly, Hypothesis 2 stated that a higher number of robots should lead to more negative ratings of robots, a higher robot anxiety, lower mind perception, and higher perceived threat.

To test Research Question 1 and Hypotheses 1 (similarity hypothesis) and 2 (number hypothesis), we conducted a multivariate analysis of variance (MANOVA) with robot number and similarity as independent variables and group perception, entitativity, perceived threat, NARS, and RAS as dependent variables. The MANOVA revealed a significant main effect of robot number on robot group perception $\left(F(9,400)=25.23, p<.001\right.$, partial $\left.\eta_{\mathrm{p}}^{2}=.36\right)$. Post hoc tests showed that there were several univariate main effects between the experimental groups. Due to the large number of significant differences, the results of the post hoc analyses will be summarized. Unsurprisingly, a single robot was less likely perceived as a group as compared to three up to ten robots (e.g., for three robots: $t(76)=7.86, p<.001, \mathrm{~d}=1.80$; for ten robots: $t(75)=10.05, p<.001, \mathrm{~d}=2.51$ ). However, two robots were not perceived as significantly different from one robot $(t(77)=2.49, p=$ $.45, \mathrm{~d}=0.56)$ in terms of group perception. Table 2 shows means and standard deviations for robot group perception for the different numbers of robots. Three robots were rated as a group to a significantly greater extent compared to one robot $(t(76)=7.86, p<.001, \mathrm{~d}=1.80)$ and two robots $(t(81)=5.55, p<.001, d=1.26)$. There were no significant differences between the group perception of three robots compared to any other number of robots ( $p$ s and effect sizes ranged 
Table 2. Descriptive Statistics Regarding

Group Perception as a Function of Robot Number

\begin{tabular}{ccc}
\hline Robot number & $M$ & $S D$ \\
\hline 1 & 1.91 & 1.54 \\
2 & 2.76 & 1.48 \\
3 & 4.63 & 1.48 \\
4 & 5.26 & 1.50 \\
5 & 4.92 & 1.59 \\
6 & 5.40 & 1.38 \\
7 & 5.19 & 1.46 \\
8 & 5.06 & 1.80 \\
9 & 5.33 & 1.71 \\
10 & 5.43 & 1.26 \\
\hline
\end{tabular}

between $p=.64, \mathrm{~d}=0.58$ for ten robots and $p=1, \mathrm{~d}=0.19$ for five robots). The same pattern of results was obtained for all other groups of robots (that is, the groups ranging from four to ten robots): Each condition differed significantly in its group perception from one and two robots, but did not significantly differ from any of the other conditions. The $p$-values for all significant differences were $p<.001$, with effect sizes ranging from $t(80)=5.55, p<.001, \mathrm{~d}=1.26$ for the difference between two and three robots, and $t(75)=10.05, p<.001, \mathrm{~d}=2.51$ for the difference between one and ten robots. The nonsignificant results between the stimuli that showed four, five, six, seven, eight, and ten robots ranged from $t(79)=2.29, p=.64, \mathrm{~d}=0.58$ for the comparison of three versus ten robots, to $p=1.000, \mathrm{~d}<=0.19$ for most of the other comparisons.

Furthermore, the MANOVA showed a significant main effect of similarity on group entitativity $\left(F(1,400)=5.32, p=.02, \eta_{\mathrm{p}}^{2}=.13\right)$. That is, highly similar robots were perceived as more entitative $(M=4.17, S E=.09)$ than robots low in similarity $(M=3.88, S E=.09, t(418)=2.31, p=$ $.02, d=0.22)$. Furthermore, we obtained a marginally significant main effect of similarity on mind attribution $\left(F(1,400)=3.86, p=.05, \eta_{\mathrm{p}}^{2}=.10\right)$. That is, highly similar robots were perceived as having less mind $(M=2.72, S E=.07)$, compared to robots low in similarity $(M=2.90, S E=$ $.06, t(418)=1.97, p=.05, \mathrm{~d}=0.20)$. Figure 3 depicts these results:

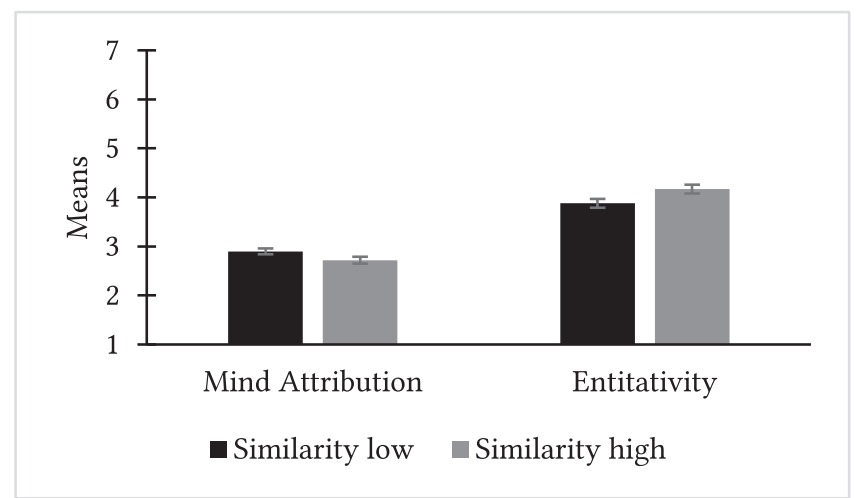

Fig. 3. Effects of similarity on mind attribution and entitativity. Error bars indicate standard errors. 
No significant effects were found for NARS, RAS, and perceived threat, neither for robot number $\left(F(9,400)=1.40, p=.18, \eta_{\mathrm{p}}^{2}=.03\right.$ for NARS, $F(9,400)=1.44, p=.17, \eta_{\mathrm{p}}^{2}=.03$ for RAS $)$ nor for similarity $\left(F(1,400)=0.82, p=.37, \eta_{\mathrm{p}}^{2}=.00\right.$ for NARS, $F(1,400)=0.16, p=69, \eta_{\mathrm{p}}^{2}=$ .00 for RAS). No interaction effects were found between robot number and similarity (all $p s>$ .18 , effect sizes ranging from $F(9,400)=0.36, p=.95, \eta_{\mathrm{p}}^{2}=.01$ for robot group perception to $F(9,400)=1.43, p=.18, \eta_{\mathrm{p}}^{2}=.03$ for mind attribution). To sum up, our first main analysis referred to Research Question 1 in showing that three robots seem to be necessary to elicit group perception. Additionally, results showed that there were no differences in perceived group entitativity with regards to the number of robots at display. Furthermore, the analysis partially confirmed Hypothesis 1 (similarity hypothesis) but did not confirm Hypothesis 2 (number hypothesis).

To make sure that the effects we found were due to changes in experimental conditions and not due to effects of covariates, we computed a MANCOVA and controlled for influences of potential covariates. Following the principle of parsimony [41], only covariates that had significantly influenced the dependent variables were included in the MANCOVA. These were robot experience, sex, age, technical acceptance and usage, whether participants were employed or not, whether their job was of a technical nature, and their marital status. When the results were controlled for covariate influences, the previously significant differences for similarity on mind attribution and perceived group entitativity did not turn out statistically significant anymore $\left(F(1,383)=2.00, p=.16, \eta_{\mathrm{p}}^{2}=\right.$ .01 for mind attribution and $F(1,383)=2.42, p=.12, \eta_{\mathrm{p}}^{2}=.01$ for entitativity). Similarity did not have a significant effect on any of the other dependent variables ( $p$ s and effect sizes ranging from $p=.30, \eta_{\mathrm{p}}^{2}=.00$ for robot group perception to $p=.78, \eta_{\mathrm{p}}^{2}=.00$ for perceived threat).

The main effect of robot number on robot group perception, however, remained significant even after controlling for the covariates $\left(F(9,383)=57.54, p<.001, \eta_{\mathrm{p}}^{2}=.37\right)$. Table 3 displays means and standard errors for robot group perception when controlled for covariates. Post hoc tests showed that the pattern of results was similar to the one found in the MANOVA. As before, being presented with one robot resulted in a significantly different group perception from three up to ten presented robots (e.g., for four robots: $t(77)=9.81, p<.001, \mathrm{~d}=2.20$; for ten robots: $t(73)=9.65, p<.001, \mathrm{~d}=2.20)$ and did not differ from two presented robots $(t(76)=2.24, p=.70, \mathrm{~d}=0.55)$. Two robots also differed in their perception as a group from all other presented numbers of robots (e.g., for three robots $t(81)=5.75, p<.001, \mathrm{~d}=1.27$ ), except from one robot $(t(76)=2.24, p=.70, \mathrm{~d}=0.55)$. All other numbers of robots (i.e., three up to ten)

Table 3. Means and Standard Errors for Robot Group Perception Controlled for Covariate Effects

\begin{tabular}{ccc}
\hline Robot number & $M$ & $S E$ \\
\hline 1 & 1.93 & 0.26 \\
2 & 2.71 & 0.24 \\
3 & 4.66 & 0.24 \\
4 & 5.37 & 0.24 \\
5 & 4.91 & 0.23 \\
6 & 5.39 & 0.24 \\
7 & 5.15 & 0.24 \\
8 & 5.15 & 0.24 \\
9 & 5.30 & 0.24 \\
10 & 5.36 & 0.25 \\
\hline
\end{tabular}


differed significantly from one and two robots with regards to group perception (all $p \mathrm{~s}<.001$ ), but did not significantly differ from each other ( $p$ s and effect sizes ranging from $p=.80, \mathrm{~d}=0.51$ for the difference between three and six robots to $p=1.00, \mathrm{~d}<=0.06$ for the difference between four robots and six robots, as well as most of the other nonsignificant differences).

Analogous to the initial results obtained from the MANOVA, no significant effects of robot group on any of the other dependent variables were found (effect sizes ranging from $\eta_{\mathrm{p}}^{2}=.03$ for entitativity $\eta_{\mathrm{p}}^{2}=.01$ for perceived threat). Furthermore, no significant interaction effects were found ( $p \mathrm{~s}$ ranging from $p=.22, \eta_{\mathrm{p}}^{2}=.03$ for mind attribution, $p=.94, \eta_{\mathrm{p}}^{2}=.01$ for robot group perception). To sum up, the findings of the analyses using MANCOVA to some extent mirrored those of the MANOVA, as the results with regards to the number of robots necessary for group perception was the same, whereas Hypothesis 2 (number hypothesis) was not empirically supported. Hypothesis 1 (similarity hypothesis) had been partially supported by the MANOVA results but was not supported by the results of the MANCOVA, which leads to the conclusion that the results for Hypothesis 1 might have been significant due to effects of covariates.

\section{DISCUSSION}

The present experiment examined the influence of robot number and similarity on robot group perception and entitativity. The experiment was motivated by two pertinent research issues. First, our work was motivated by the fact that, to date, the perception of robots as (social) groups has not been systematically tested, despite the tacit assumption that robots-and large numbers of them-will cooperate with us in teams or assist us in homes, schools, or care settings. In the future, people are likely to be confronted with groups of robots at the same time. From research about human groups, however, we know that whether an individual is seen as part of a social group influences behavior toward the individual $[4,7-10,19]$. Consequently, for the prediction and understanding of user reactions toward a given number of robots, it is important to shed light on the conditions under which they are in fact perceived as a (social) group. Second, existing research on the topic based on the implicit assumption that the perception of (groups of) robots and (groups of) humans mostly follows the same psychological principles, even though the inconclusive state-of-the-art literature suggests that there might be major differences. Our results showed that a rather conservative approach toward the adoption of knowledge on groups of humans for groups of robots without previous testing is justified, as we observed a difference in the perception of human versus robot groups. Even though our methodological approach, which was a merely visual presentation without actual interaction, might be different in comparison to the approaches used in some of the other studies, our results should be seen as a call for action to carefully test the psychological knowledge and mechanisms from human context before transferring those to the robot context.

Social groups of humans consist, per definition, of two or more human beings $[25,26]$ who share characteristics (such as a common goal, norms that determine behavior, etc.). This is not in line with what we observed in the context of robot groups: The results of our analyses imply that two robots might not be sufficient in leading to group perception, regardless of whether they were high or low in similarity. Even two very similar robots were not perceived as a group but seemed to be perceived as being two distinct entities; that is, similarity did not influence group perception. This holds true regardless of age, sex, occupation, technical experience, technical affinity, or robot experience of those who evaluate the robots. The results implies that the studies aiming at examining robot groups must start testing the very basics: by finding out what makes a group of robots a group. However, our results do not only show differences, but also potential similarities between groups of robots and groups of humans, in that a higher similarity led to a higher 
perceived entitativity, which is in line with the claims of Reference [23]. These significant differences vanished, though, when we controlled for potential covariates. This indicates that the differences in entitativity might be due to differences in characteristics of the sample. The same holds true for the differences we found for mind attribution: Higher similarity of the robots led to a lower mind attribution toward robots in general, but the effect also lost its significance when covariates were controlled for. This leads to the assumption that the differential perception of robots with regards to mind attribution and entitativity might rather exist due to demographic differences of the sample than due to systematic differences created by our experimental manipulation. Rather unexpectedly, a higher number of robots did not lead to higher perceived threat, even when the robots were depicted as highly similar. This could be due to our stimulus material: We used pictures of the NAO robot, a rather friendly looking, small humanoid. Even though we controlled for prior experience with NAO to rule out effects of familiarity, this robot still appears less threatening than other robot types. Accordingly, it is likely that results with regards to perceived threat might differ when using another robot prototype, in that a higher number of robots would expectedly lead to a higher perceived threat.

Based on our initial empirical evidence, we have several recommendations for future research: First, prospective research should try to replicate the findings with other robot prototypes to test for generalizability. Second, one could argue that the robot stimuli in the condition with low similarity nevertheless appeared already quite similar, as they depicted the same robot just in slightly different coloring and showing different poses. We therefore recommend replicating the experiment with groups of robots that show less similarity than the ones we used, using variously colored robots or different robot types, which also vary in size, for example. This would also take into account the possibility that participants might have perceived the similar robot groups as military formations. However, in Germany, where the experiment was conducted, military parades are very rare. Thus, the concept of military formations would not necessarily be cognitively accessible for participants. Additionally, due to participants' alleged unfamiliarity with the military, being confronted with a group of robots that could potentially resemble a military formation would presumably evoke a threat response. However, with our measure of perceived threat it was possible to take this into account. Our results did not show any differences with regards to perceived threat between any of the presented groups, which hints at the fact that the similar robots were not perceived as a military formation. Nonetheless, future studies should use less-similar robots, as described above, to rule out any possibility of a perception as a military formation. Third, future studies might want to include comparable human control conditions. We chose to concentrate on the perception of only robots and built upon what is known from the literature on the perception of human groups. This was done especially as we already had a very high number of experimental groups. Therefore, we chose to focus on the robots. However, to strengthen the explanatory power of the results, it would be recommendable to include groups of humans as a reference. This would give an even better insight into the differences between the perception of robots and human beings. A suggestion to bring it to a manageable number of robot groups could be to include the seemingly crucial numbers of one to three and add four to be sure, and then only include numbers of eight, nine, and ten, as the numbers in between did not seem to make a difference. In addition, we recommend using a higher sample size to improve statistical power. With our sample size, it is likely that small effects do not reach statistical significance. A higher sample size would increase statistical power, therefore making it more likely for middle-sized or small effects to reach significance. Fourth, our experiment examined robot group perception using pictures and it yet has to be tested whether the results generalize to scenarios that include actual human-robot interaction. This is especially relevant, as previous research on robot groups (e.g., References [12, $15,30,31])$ used different methodological approaches when examining robot groups; that is, video 
material or real-life interaction. The study by Lickel [23], though, uses a text-based approach when examining groups of human beings. If actual human-robot interaction cannot be realized because of a lack of available robots, it would be advisable to at least include video material when introducing the robots in a follow-up study. Generally, processing static images is vastly different from actual, embodied human-robot interaction, and, therefore, might lead to different results. Thus, using a video-based scenario would come closer to actual HRI and, consequently, would increase external validity. On a related note, it is important to consider the fact that Lickel [23] described pre-existing groups of humans, such as members of a sports team, and, by that, automatically provided a context. Our approach, which consisted of grouping the robots together in a visual manner, did not provide context, and is, consequently, less true-to-life. Even though groups of robots comparable to the groups by Lickel [23] do not yet exist, it might be recommendable for future studies to create a more comparable context as used by Reference [23] for the robot groups presented; examples might be rescue robots during a mission or experimental robots for usage in studies. Another possible suggestion for improvement would be to present the robots as sharing a physical space, instead of presenting them in front of a blank background. Moreover, it might be recommendable to use another operationalization of similarity. In the present research, we presented identical versus non-identical robots. To reduce the likelihood that identical robots would remind participants of military formations, future studies could use robot stimuli that vary in color or type. Fifth, our findings also highlight the importance of considering covariates: the results changed when taking them into account. We thus recommend to systematically examine the influence of demographic variables and personality traits on robot group perception, e.g., by comparing samples with certain demographic characteristics. Our experiment is just a first step into examining the basic influence factors of robot group perception and robot group entitativity. For future research it would be interesting to go beyond mere robot number and test and manipulate other influence factors on group entitativity, such as a shared goal, for example (see References [42] and [43] for picture-based manipulations of shared goals in human context). Finally, a well-known problem of online studies is the fact that it is beyond the researchers' control if participants really concentrate on the study at hand or do something else along the way. Thus, it might be beneficial to replicate the study using a lab-based approach.

Notwithstanding the limitations, the present experiment raises important issues to be considered when designing future experiments aiming at examining robot groups. Most importantly, our work lends support to the fact that more basic psychological research needs to be realized to understand the boundary conditions of perceiving even nonhuman entities as groups. This is particularly relevant considering the many use cases for robots that rest on the idea that multiple robots will co-inhabit our social environment. Our findings show that while it takes two humans to form a social group, it might take more than just two robots to elicit group perception in robots. Based on this, we suggest that future studies use at least three robots when aiming to test psychological principles and mechanisms pertaining to robot groups and include measures to test whether the given number of robots was perceived as a group or a number of individuals instead. Our results imply the necessity to question whether basic principles taken from social psychological group research can simply be transferred and adapted to social robotics. The present findings suggest that taking an empirical approach to gather evidence before generalizing knowledge from one field to the other might be fruitful. Our work contributes to the understanding of the extent to which knowledge from human groups applies to robot groups and gives advice for the design of future studies examining these very principles. Therefore, the experiment adds to the fields of social psychology as well as HRI by providing insights into the basic psychological principles of robot group perception. Hopefully, our results inspire future studies to help systemize the knowledge 
we have about groups of human beings for robot groups as well and motivate other researchers to test social psychological paradigms in the robot context before applying them in their research.

\section{ACKNOWLEDGMENTS}

We report how we determined our sample size, all data exclusions (if any), all manipulations, and all measures in the study. Ethics approval was granted by the Ethics Commission of Bielefeld University (2019-166). We would like to thank Jasmin Bernotat, Julia Stapels, and Angelika Penner for their feedback on previous versions of this manuscript.

\section{REFERENCES}

[1] International Federation of Robotics Statistical Department. 2012. World Robotics: Service Robots. Retrieved from http://www.ifr.org/service-robots/statistics/.

[2] Ulrich Reiser, Christian Connette, Jan Fischer, Jens Kubacki, Alexander Bubeck, Florian Weisshardt, Theo Jacobs, Christopher Parlitz, Martin Hägele, and Alexander Verl. 2009. Care-O-bot ${ }^{\circledR}$ 3-creating a product vision for service robot applications by integrating design and technology. In Proceedings of the 22nd International Conference on Intelligent Robots and Systems (IROS'09). IEEE/RSJ, 1992-1998. DOI : https://doi.org/10.1109/IROS.2009.5354526

[3] Claire Huijnen, Atta Badii, Herjan van den Heuvel, Praminda Caleb-Solly, and Daniel Thiemert. 2011. "Maybe it becomes a buddy, but do not call it a robot"-Seamless cooperation between companion robotics and smart homes. In Proceedings of the 2nd International Joint Conference on Ambient Intelligence. 324-329. DOI : https://doi.org/10.1007/ 978-3-642-25167-2_44

[4] Christopher L. Aberson, Michael Healy, and Victoria Romero. 2000. Ingroup bias and self-esteem: A meta-analysis. Personal. Soc. Psychol. Rev. 4, 2 (2000), 157-173. DOI: https://doi.org/10.1207/S15327957PSPR0402_04

[5] Harold H. Kelley and J. W. Thibaut. 1978. Interpersonal Relations. Wiley, New York.

[6] Michael A. Hogg. 2000. Subjective uncertainty reduction through self-categorization: A motivational theory of social identity processes. Euro. Rev. Soc. Psychol. 11, 1 (2011), 223-255. DOI : http://dx.doi.org/10.1080/14792772043000040

[7] Henri Tajfel, M. G. Billig, R. P. Bundy, and Claude Flament. 1971. Social categorization and intergroup behaviour. Euro. J. Soc. Psychol. 1, 2 (1971), 149-178. DOI : 10.1002/ejsp.2420010202

[8] Marilyn B. Brewer and Joseph G. Weber. 1994. Self-evaluation effects of interpersonal versus intergroup social comparison. 7. Personal. Soc. Psychol. 66, 2 (1994), 268-275. DOI:http://dx.doi.org/10.1037/0022-3514.66.2. 268

[9] Rupert Brown. 1988. Group Processes: Dynamics within and between Groups. Blackwell, Oxford, UK.

[10] David L. Hamilton and Stephen J. Sherman. 1996. Perceiving persons and groups. Psychol. Rev. 103, 2 (1996), $336-355$. DOI : http://dx.doi.org/10.1037/0033-295X.103.2.336

[11] Clay Beckner, Peter Racz, Jennifer Hay, Jürgen Brandstetter, and Christoph Bartneck. 2016. Participants conform to humans but not to humanoid robots in an English past tense formation task. F. Lang. Soc. Psychol. 35, 2 (2016), 158-179. DOI : https://doi.org/10.1177/0261927X15584682

[12] Jürgen Brandstetter, Peter Racz, Clay Beckner, Eduardo B. Sandoval, Jenniger Hay, and Christoph Bartneck. 2014. A peer pressure experiment: Recreation of the Asch conformity experiment with robots. In Proceedings of the International Conference on Intelligent Robots and Systems (IROS'14). IEEE/RSJ, 1335-1340. DOI : https://doi.org/10.1109/IROS. 2014.6942730

[13] Nicole Salomons, Michael van der Linden, Sarah Strohkorb Sebo, and Brian Scassellati. 2018. Humans conform to robots: Disambiguating trust, truth, and conformity. In Proceedings of the 13th International Conference on HumanRobot Interaction (HRI'18). ACM/IEEE, 187-195. DOI : https://doi.org/10.1145/3171221.3171282

[14] Marlena R. Fraune, Satoru Kawakami, Selma Šabanović, P. Ravindra de Silva, and Michio Okada. 2015. Three’s company, or a crowd?: The effects of robot number and behavior on HRI in Japan and the USA. In Proceedings of the 12th Annual Robotics: Science and Systems Conference. DOI : http://dx.doi.org/10.15607/RSS.2015.XI.033

[15] Marlena R. Fraune, Yusaku Nishiwaki, Selma Sabanović, Eliot Smith, and Michio Okada. 2017. Threatening flocks and mindful snowflakes: How group entitativity affects perceptions of robots. In Proceedings of the 12th International Conference on Human-Robot Interaction (HRI'17). ACM/IEEE, 205-213. DOI : http://dx.doi.org/10.1145/2909824.3020248

[16] Marlena R. Fraune, Steve Sherrin, Selma Šbanović, and Eliot Smith. 2015. Rabble of robots effects: Number and form of robots modulates attitudes, emotions, and stereotypes. In Proceedings of the 10th Annual International Conference on Human-Robot Interaction (HRI'15), ACM/IEEE, 109-116. DOI : https://doi.org/10.1145/2696454.2696483

[17] Dieta Kuchenbrandt, Friederike Eyssel, Simon Bobinger, and Maria Neufeld. 2011. Minimal group-maximal effect? Evaluation and anthropomorphization of the humanoid robot NAO. Lect. Notes Comput. Sci. 7072 (2011), $104-113$. DOI : $10.1007 / 978-3-642-25504-5 \_11$ 
[18] Dieta Kuchenbrandt, Friederike Eyssel, Simon Bobinger, and Maria Neufeld. 2013. When a robot's group membership matters. Int. J. Soc. Robot. 5, 3 (2013), 409-417. DOI : https://doi.org/10.1007/s12369-013-0197-8

[19] Friederike Eyssel and Steve Loughnan. 2013. "It don't matter if you're black or white"? Effects of robot appearance and user prejudice on evaluations of a newly developed robot companion. Lect. Notes Artif. Intell. 8239 (2013), 422-431. DOI : https://doi.org/10.1007/978-3-319-02675-6_42c

[20] Richard P. McGlynn, Deborah J. Harding, and Jaqueline L. Cottle. 2009. Individual-group discontinuity in groupindividual interactions: Does size matter? Group Proc. Intergr. Relat. 12, 1 (2009), 129-143. DOI: https://doi.org/10. 1177/1368430208098781

[21] Brian P. Meier and Verlin P. Hinsz. 2004. A comparison of human aggression committed by groups and individuals: An interindividual-intergroup discontinuity. f. Exper. Soc. Psychol. 40, 4 (2004), 551-559. DOI : https://doi.org/10.1016/ j.jesp.2003.11.002

[22] Tim Wildschut, Brad Pinter, Jack L. Vevea, Chester A. Insko, and John Schopler. 2003. Beyond the group mind: A quantitative review of the interindividual-intergroup discontinuity effect. Psychol. Bull. 129, 5 (2003), 698-722. DOI : 10 1037/0033-2909.129.5.698

[23] Brian Lickel, David L. Hamilton, Grazya Wieczorkowska, Amy Lewis, Steven J. Sherman, and A. Neville Uhles. 2000. Varieties of groups and the perception of group entitativity. F. Personal. Soc. Psychol. 78, 2 (2000), 223-246. DOI : 10. 1037//0022-3514.78.2.223

[24] Donald T. Campbell. 1958. Common fate, similarity, and other indices of the status of aggregates of persons as social entities. Behav. Sci. 3, 1 (1958), 14-25. DOI : 10.1002/bs.3830030103.

[25] John C. Turner. 1982. Towards a cognitive redefinition of the social group. In Social Identity and Intergroup Relations, Henri Tajfel (Ed.). Cambridge University Press and Edition de la Maison des Sciences de l'Homme, Cambridge/Paris, 15-49.

[26] Donelson R. Forsyth. 2006. Group Dynamics (4th ed.). Thomson Wadsworth Publishing, Belmont, CA.

[27] Georg Simmel. 1902. The number of members as determining the sociological form of the group. I. Amer. F. Sociol. 8, 1 (1902), 1-46. DOI : http://dx.doi.org/10.1086/211115

[28] Georg Simmel. 1902. The number of members as determining the sociological form of the group. II. Amer. F. Sociol. 8, 2 (1902), 158-196. DOI : http://dx.doi.org/10.1086/211128

[29] John James. 1951. A preliminary study of the size determinant in small group interaction. Amer. Sociol. Rev. 16, 4 (1951), 474-477. DOI: $10.2307 / 2088278$

[30] Wan-Ling Chang, Jeremy P. White, Johyun Park, Anny Holm, and Selma Šabanović. 2012. The effect of group size on people's attitudes and cooperative behaviors toward robots in interactive gameplay. In Proceedings of the 21 st International Symposium on Robot and Human Interactive Communication (RO-MAN'12). IEEE, 845-850. DOI : https: //doi.org/10.1109/ROMAN.2012.6343857

[31] Ahsan Nawroj, Maria Toneva, Henny Admoni, and Brian Scassellati. 2014. An exploration of social grouping in robots: Effects of behavioral mimicry, appearance, and eyegaze. In Proceedings of the 36th Annual Conference of the Cognitive Science Society (CogSci'14). 1060-1065.

[32] Robert J. Rydell and Allen R. McConnell. 2005. Perceptions of entitativity and attitude change. Pers. and Soc. Psychol. Bull. 31, 1 (2005), 99-110. DOI : https://doi.org/10.1177/0146167204271316

[33] David L. Hamilton, Jaqueline M. Chen, and Nate Way. 2011. Dynamic aspects of entitativity. In Social Cognition, Social Identity, and Intergroup Relations: A Festschrift in Honor of Marilynn B. Brewer, Roderick M. Kramer, Geoffrey J. Leonardelli and Robert W. Livingston (Eds.). Psychology Press, 36-60.

[34] Carey K. Morewedge, Jesse J. Chandler, Robert Smith, Norbert Schwarz, and Jonathan Schooler. 2013. Lost in the crowd: Entitative group membership reduces mind attribution. Consc. $\operatorname{cog} n$. 22, 4 (2013), 1195-1205. DOI : https://doi. org/10.1016/j.concog.2013.08.002

[35] Julie Spencer-Rodgers, Melissa J. Williams, David L. Hamilton, Kaiping Peng and Lei Wang. 2007. Culture and group perception: Dispositional and stereotypic inferences about novel and national groups. f. Personal. Soc. Psychol. 93, 4 (2007), 525-543. DOI : 10.1037/0022-3514.93.4.525

[36] Franz J. Neyer, Juliane Felber, and Claudia Gebhardt. 2012. [Development and validation of a short-scale to measure acceptance and usage of new technology]; Entwicklung und Validierung einer Kurzskala zur Erfassung von Technikbereitschaft. Diagnostica 58, 2 (2012), 87-99. DOI : https://doi.org/10.1026/0012-1924/a000067

[37] Tatsuya Nomura, Takayuki Kanda, Tomohiro Sizuki, and Kennsuke Kato. 2004. Psychology in human-robot communication: An attempt through investigation of negative attitudes and anxiety toward robots. In Proceedings of the 13th International Symposium on Robot and Human Interactive Communication (RO-MAN'04). IEEE, 35-40. DOI : http://dx.doi.org/10.1109/ROMAN.2004.1374726

[38] Tatsuya Nomura, Tomohiro Suzuki, Takayuki Kanda, and Kennsuke Kato. 2006. Measurement of anxiety toward robots. In Proceedings of the 15th International Symposium on Robot and Human Interactive Communication (ROMAN'06). IEEE, 372-377. DOI : https://doi.org/10.1109/ROMAN.2006.314462 
[39] Heather M. Gray, Kurt Gray, and Daniel M. Wegner. 2007. Dimensions of mind perception. Science 315, 5812 (Feb. 2007), 619-619. DOI : https://doi.org/10.1126/science.1134475

[40] Natalia Reich-Stiebert and Friederike Eyssel. 2015. Learning with educational companion Robots? Toward attitudes on education robots, predictors of attitudes, and application potentials for education robots. Int. F. Soc. Robot. 7, 5 (2015), 875-888. DOI : https://doi.org/10.1007/s12369-015-0308-9

[41] Barbara G. Tabachnick and Linda S. Fidell. 2013. Using Multivariate Statistics (6th ed.). Pearson Education, Boston, MA.

[42] Arne Sjöström, Mario Gollwitzer. 2015. Displaced revenge: Can revenge taste "sweet" if it aims at a different target? f. Exper. Soc. Psychol. 56 (2014), 191-202. DOI : https://doi.org/10.1016/j.jesp.2014.09.016

[43] Maria Plötner, Harriet Over, Malinda Carpenter, and Michael Tomasello. 2016. What is a group? Young children's perceptions of different types of groups and group entitativity. PloS One 11, 3 (2016), e0152001. DOI : 10.1371/journal. pone. 0152001

Received May 2018; revised February 2020; accepted April 2020 